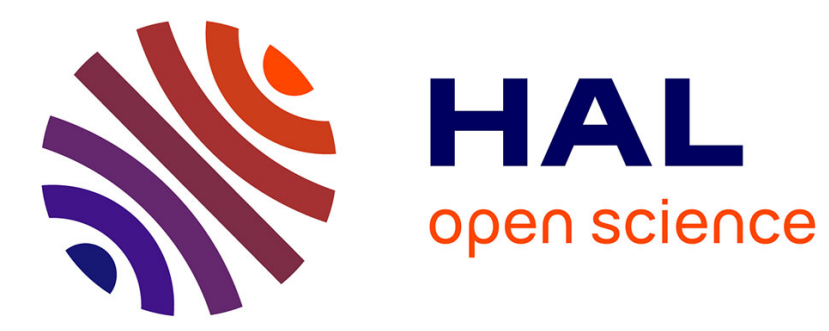

\title{
Effective properties of elastic periodic composite media with fibers
}

\author{
Guy Bonnet
}

\section{To cite this version:}

Guy Bonnet. Effective properties of elastic periodic composite media with fibers. Journal of the Mechanics and Physics of Solids, 2007, 55 (5), pp.881-899. 10.1016/j.jmps.2006.11.007 . hal-00691103

\section{HAL Id: hal-00691103 \\ https://hal.science/hal-00691103}

Submitted on 17 Jan 2016

HAL is a multi-disciplinary open access archive for the deposit and dissemination of scientific research documents, whether they are published or not. The documents may come from teaching and research institutions in France or abroad, or from public or private research centers.
L'archive ouverte pluridisciplinaire HAL, est destinée au dépôt et à la diffusion de documents scientifiques de niveau recherche, publiés ou non, émanant des établissements d'enseignement et de recherche français ou étrangers, des laboratoires publics ou privés. 


\title{
Effective properties of elastic periodic composite media with fibers
}

\author{
G. Bonnet* \\ Laboratoire de Mécanique, Université de Marne la Vallée, EA 2545, Institut Navier, \\ 5 boulevard Descartes, 77454 Marne la Vallée cedex, France
}

\begin{abstract}
A series solution to obtain the effective properties of some elastic composites media having periodically located heterogeneities is described. The method uses the classical expansion along Neuman series of the solution of the periodic elasticity problem in Fourier space, based on the Green's tensor, and exact expressions of factors depending on the shape of the inclusions. Some properties of convergence of the solution are presented, more specifically concerning the elasticity tensor of the reference medium, showing that the convergence occurs even for empty fibers. The solution is extended for rigid inclusions. A comparison is made with previous exact solutions for a fiber composite made of cylindrical fibers with circular cross-sections and with previous estimates. Different examples are presented for new situations concerning the study of fiber composites: composites with elliptic cross-sections and multi-phase fibrous composites.
\end{abstract}

Keywords: Elasticity; Composites; Fourier transform; Neuman series; Effective properties

\section{Introduction}

Determining the elastic "effective" properties of a composite multi-phase material has been the subject of numerous works. When the microstructure is periodic, a rigorous procedure can be described to obtain the effective properties, if a "localization" problem is solved on a period. Approximations are used to compute (Cohen, 2004; Luciano and

\footnotetext{
*Tel.: + 3301609572 20; fax: + 33160957799 .

E-mail address: bonnet@univ-mlv.fr.
} 
Barbero, 1994; Adams and Doner, 1967; Segurado and Llorca, 2002) or to bound (Hashin and Shtrikman, 1962) the effective properties, including finite-element solutions (Gusev, 1997), boundary-element solutions (Helsing, 1995) or the use of Fourier transforms (Michel et al., 1999; Moulinec and Suquet, 1994), which allows to sum a Neuman series, along the lines of a method which was introduced for composite conductors by Brown (1955). Few exact solutions exist, except for periodic arrays of spheres (Sangani, 1987) or fiber-reinforced composites in the case of specific geometries (Kantor and Bergman, 1982; Guinovart-Diaz et al., 2001). In this last case, the solution involves, however, the inversion of an infinite linear system. Iwakuma and Nemat-Nasser (1983) obtained estimates of the mean properties of a periodic arrangement of elliptical fibers or ellipsoidal inclusions.

The purpose of the paper is to analyze solutions based on the classical Neuman series, to show that the terms of the series can be obtained by a closed form recurrence process for some microstructures, even for rigid or void inclusions, to analyze the convergence of the solution and to show the application to new situations.

In a first step, the localization and homogenization processes are described. The solution using the Neuman series is then introduced and it is shown that an exact solution can be obtained for specific microstructures. A comparison is made with other exact solutions and the convergence of the solution is studied, by looking for the optimized "reference medium" related to a given microstructure. The cases of extremal values for the elastic properties of the inclusion are then studied: empty inclusions and rigid inclusions. Finally examples of applications to new situations are presented: three-phase periodic material and fibers with an elliptic cross-section.

\section{Description of the localization and homogenization processes}

An elastic periodic composite material is described by its elastic properties on a volumic period $\Omega$. This period is a parallelepiped whose length in the direction $i$ is $\Lambda_{i}$. The elastic properties are assumed to be constant within the volume $V_{\alpha}$ characterizing each phase $\alpha$. So each phase is characterized by an elastic tensor having the components $L_{i j k l(\alpha)}$. The asymptotic expansion method allows the construction of the displacement field at the scale of the microstructure by looking for an expansion in powers of a small parameter $\varepsilon=l / L$, where $l$ is a length which is characteristic of the microstructure and $L$ is a length which is characteristic of the structure.

The asymptotic method used to build the localization process uses a series representation of the displacement field $\mathbf{u}$ of the form $\mathbf{u}=\mathbf{u}^{(0)}(\mathbf{y}, \mathbf{x})+\varepsilon \mathbf{u}^{(1)}(\mathbf{y}, \mathbf{x})+\varepsilon^{2} \mathbf{u}^{(2)}$ $(\mathbf{y}, \mathbf{x})+\cdots$, where $\mathbf{y}$ characterizes the "macroscopic" position of the period within the structure and $\mathbf{x}$ characterizes the "microscopic" position of a point within the period.

When using an adequate "scaling" of the elastic properties, different kinds of solutions can be obtained (Boutin, 1996), but usually, only the leading terms are kept (Auriault and Bonnet, 1985; Bensoussan et al., 1978; Sanchez-Palencia, 1974, 1980) and the following results are obtained:

(A) $\mathbf{u}^{(0)}$ is only a function of $\mathbf{y}$. The associated strain tensor is denoted by $\mathbf{e}_{y}\left(\mathbf{u}^{(0)}\right)$ and corresponds to the macroscopic deformation tensor $\mathbf{E}$.

(B) $\mathbf{u}^{(1)}$ is the periodic solution of the PDE problem on $\Omega$

$$
\operatorname{Div}_{x}\left(\mathbf{L} \cdot\left(\mathbf{e}_{x}\left(\mathbf{u}^{(1)}\right)+\mathbf{e}_{y}\left(\mathbf{u}^{(0)}\right)\right)\right)=0,
$$




$$
\begin{aligned}
& {\left[\mathbf{L} .\left(\mathbf{e}_{x}\left(\mathbf{u}^{(1)}\right)+\mathbf{e}_{y}\left(\mathbf{u}^{(0)}\right)\right) \cdot \mathbf{N}\right]_{\Gamma}=0,} \\
& {\left[\mathbf{u}^{(1)}\right]_{\Gamma}=0,}
\end{aligned}
$$

where $\mathbf{u}^{(1)}$ is a periodic function of the local variable $\mathbf{x}$; index $x$ means that the derivation is performed on the local variable $\mathbf{x}$ and similarly for $y$. $\mathbf{L}$ is the elasticity tensor at the local position $\mathbf{x} . \Gamma$ denotes the surface corresponding to the interfaces between phases. The quantity $[f]$ is related to the discontinuity of $f$ along the interface $\Gamma$ between inclusions and matrix.

(C) The solution $\mathbf{u}^{(1)}$ of problems (1) (3) is linearly related to components of the macroscopic deformation tensor $\mathbf{E}=\mathbf{e}_{y}\left(\mathbf{u}^{(0)}\right)$ by a relation of the type:

$$
u_{i}^{(1)}={ }_{(j k)} \xi_{i} \cdot E_{j k}\left(\mathbf{u}^{(0)}\right)+u_{i}^{\prime}(\mathbf{y}),
$$

where ${ }_{j k} \xi_{i}$ are the components of a localization tensor whose components are the particular solutions of problems (1) (3) for macroscopic tensors given by $E_{p q}=\delta_{p j} . \delta_{q k} . \delta_{p k}$ are the components of the Kronecker tensor.

(D) The components of the "effective" elasticity tensor $\mathbf{L}^{\text {eff }}$ are obtained from the localization tensor by

$$
L_{i j k h}^{\mathrm{eff}}=\left\langle L_{i j l m} e_{x l m}\left((j k) \xi_{i}\right)+L_{i j k h}\right\rangle,
$$

where the brackets denote the volumic mean over $\Omega$ and the deformations are computed from the indices $i$ of the components ${ }_{(j k)} \xi_{i}$.

The steps (A)-(C) constitute the localization process (in fact as much localization processes as different values of the components of the macroscopic deformation). Step (D) constitutes the homogenization process.

\section{Solution of the localization problem}

The solution of the localization problem is now sought in the case of a matrix-inclusions material.

The local elasticity tensor $\mathbf{L}$ can therefore be written as

$$
\mathbf{L}(\mathbf{x})=\Sigma_{\alpha} I_{\alpha}(\mathbf{x}) \cdot \mathbf{L}_{\alpha},
$$

where $\mathbf{L}_{\alpha}$ is the elasticity tensor of the phase $\alpha$ and $I_{\alpha}$ is the characteristic function describing the volume of the phase $\alpha$.

In the following, $\mathbf{e}_{x}\left(\mathbf{u}^{(1)}\right)$ will be denoted by $\mathbf{e}^{*}$ and the sum $\mathbf{e}^{*}+\mathbf{E}$ by $\mathbf{e}$.

The solution is now sought by using the classical expansion into the Neuman series and the Green's tensor for an isotropic reference material along the following lines:

Introducing a constant elastic reference tensor $\mathbf{L}_{\mathbf{0}}$ within (1) leads to:

$$
\operatorname{Div}\left(\mathbf{L}_{0} \cdot \mathbf{e}^{*}+\tau\right)=0,
$$

where the "polarization tensor" $\tau$ is given by:

$$
\tau=\delta \mathbf{L} \cdot\left(\mathbf{e}^{*}+\mathbf{E}\right)+\mathbf{L}_{0} \cdot \mathbf{E}
$$

and

$$
\delta \mathbf{L}(\mathbf{x})=\mathbf{L}(\mathbf{x})-\mathbf{L}_{0} .
$$


The solution of equation (6) can be expressed as the solution of the following integral equation:

$$
\mathbf{e}(\mathbf{x})=\mathbf{E}-\boldsymbol{\Gamma}_{0} *(\delta \mathbf{L} . \mathbf{e}),
$$

where the star denotes the spatial convolution product and $\boldsymbol{\Gamma}_{0}$ is the periodic fourth-order Green's operator for the deformation computed by using the reference elasticity tensor $\mathbf{L}_{0}$.

The solution of that integral equation is expressed classically by using the Neuman series (Milton, 2002):

$$
\mathbf{e}(\mathbf{x})=\left[I-\boldsymbol{\Gamma}_{0} * \delta \mathbf{L}+\boldsymbol{\Gamma}_{0} * \delta \mathbf{L} .\left(\boldsymbol{\Gamma}_{0} * \delta \mathbf{L}\right)-\cdots\right] \mathbf{E} .
$$

A discussion of the properties of such a series can be found in Milton (2002). A current way to compute the series in $(10)$ at the order $(i+1)$ is to use the equivalent explicit recurrence process starting from $\mathbf{e}^{1}=\mathbf{E}$ :

$$
\mathbf{e}^{i+1}=\mathbf{E}-\Gamma^{0} *\left(\delta \mathbf{L} \cdot \mathbf{e}^{i}\right) .
$$

Michel et al. (1999) noticed that the deformation Green's tensor has the property:

$$
\Gamma^{0} *\left(\mathbf{L}_{0} \cdot \mathbf{e}\right)=\mathbf{e}-\mathbf{E} .
$$

The iteration process can then be written in the equivalent form:

$$
\mathrm{e}^{i+1}=\mathrm{e}^{i}-\Gamma^{0} *\left(\text { L. } \mathrm{e}^{i}\right) .
$$

Both forms (11) or (13) are equivalent, as it can be checked by using either one of these forms.

In the following, the discrete Fourier transform $\mathbf{e}\left(\mathbf{k}_{(n)}\right)$ of $\mathbf{e}$ is used, where $\mathbf{k}_{(n)}$ $(n=1 \ldots \infty)$ are the discrete wave vectors arranged along a discrete network having a period 2. $\pi / \Lambda_{l}$ in the direction $l(l=1-3$, or $l=1-2$ for plane problems).

The properties of the Fourier transform lead to

$$
\mathrm{e}^{i+1}\left(\mathbf{k}_{(n)}\right)=\mathrm{e}^{i}\left(\mathbf{k}_{(n)}\right)-\boldsymbol{\Gamma}^{0}\left(\mathbf{k}_{(n)}\right) \cdot \boldsymbol{\sigma}^{i}\left(\mathbf{k}_{(n)}\right),
$$

where

$$
\boldsymbol{\sigma}^{i}(\mathbf{x})=\mathbf{L}(\mathbf{x}) \cdot \mathbf{e}^{i}(\mathbf{x}) .
$$

$\boldsymbol{\sigma}^{i}$ is the local stress tensor computed at the step $i$.

The numerical solution using (14) and (15) described by Michel et al. (1999) is based on the fact that the product $\mathbf{L} \cdot \mathbf{e}^{i}$ is local within the real space, whereas the product $\Gamma^{0}\left(\mathbf{k}_{(n)}\right) \cdot \boldsymbol{\sigma}^{i}\left(\mathbf{k}_{(n)}\right)$ is local within the space of Fourier transforms. The numerical procedure described by Michel et al. (1999) uses therefore a description of the elasticity tensor within the real space. For many inclusion-matrix problems, such a description is only approximated. As an example, if cylindrical fibers are concerned, the cross-section of the fiber must be described within the real space by using a regular mesh, which approximates the form of the section by squared elements.

From another point of view, exact expressions exist for the Fourier transforms of the characteristic functions $I_{\alpha}$ for some kinds of inclusions. These Fourier transforms denoted thereafter "shape coefficients" are the basis of the approximation of the elastic properties of elastic media obtained by Nemat-Nasser and Hori (1999). It leads to exact values of the Fourier transform of the elasticity tensor $\mathbf{L}$. 
Let us introduce the "shape coefficients" of the volume $V_{\alpha}$ of a given phase $\alpha$ defined by

$$
I_{\alpha}\left(\mathbf{k}_{(n)}\right)=\int_{V_{\alpha}} \mathrm{e}^{\mathrm{i} \mathbf{k}_{(n)} \cdot \mathbf{x}} \mathrm{d} V
$$

The Fourier transform of $\mathbf{L}(\mathbf{x})$ is given by

$$
\mathbf{L}\left(\mathbf{k}_{(n)}\right)=\sum_{\alpha} \mathbf{L}_{\alpha} I_{\alpha}\left(\mathbf{k}_{(n)}\right) \text {. }
$$

The recurrence relation within the Fourier space is now given by

$$
\mathbf{e}^{i+1}\left(\mathbf{k}_{(n)}\right)=\mathbf{e}^{i}\left(\mathbf{k}_{(n)}\right)-\Gamma^{0}\left(\mathbf{k}_{(n)}\right) \cdot \sum_{\alpha} \mathbf{L}_{\alpha}\left(I_{\alpha} * \mathbf{e}^{i}\right)\left(\mathbf{k}_{(n)}\right) .
$$

This expression gives an exact recurrence relation as soon as the shape coefficients $I_{\alpha}\left(\mathbf{k}_{(n)}\right)$ are given by closed form expressions, if the convolution product can be computed exactly. The convolution product $\left(I_{\alpha} * \mathbf{e}^{i}\right)\left(\mathbf{k}_{(n)}\right)$ can be obtained as the following limit:

$$
\left(I_{\alpha} * \mathbf{e}^{i}\right)\left(\mathbf{k}_{(n)}\right)=\lim _{N \rightarrow \infty} I_{\alpha} * \mathbf{e}^{i}\left(\mathbf{k}_{(n)}, N\right),
$$

where $I_{\alpha} * \mathbf{e}^{i}\left(\mathbf{k}_{(n)}, N\right)$ is the finite-convolution product:

$$
I_{\alpha} * \mathbf{e}^{i}\left(\mathbf{k}_{(n)}, N\right)=\Sigma_{\mathbf{k}_{(m)}} I_{\alpha}\left(\mathbf{k}_{(n)}-\mathbf{k}_{(m)}\right) \cdot \mathbf{e}^{i}\left(\mathbf{k}_{(m)}\right) .
$$

The summation being performed on the vectors $\mathbf{k}_{(m)}$ in finite number having components $\left|k_{l(m)}\right| \leqslant 2 \pi N / \Lambda_{l}$.

The finite-convolution product can now be computed exactly by using the finite-fast Fourier transform of $I_{\alpha}$ and $\mathbf{e}_{y}^{i}$.

The previous developments make it necessary to compute the shape coefficients defined by (16).

The shape coefficients include those of the matrix: $\alpha=m$ and those of the inclusions: $\alpha \neq m$.

It is clear from (16) that

For $\mathbf{k}_{(n)} \neq 0$ :

$$
\sum_{\alpha} I_{\alpha}\left[\mathbf{k}_{(n)}\right]=0
$$

while for $\mathbf{k}_{(n)}=0, I_{\alpha}=V_{\alpha}$, where $V_{\alpha}$ is the volume of the phase $\alpha$ within the period.

It means that it is sufficient to compute all $I_{\alpha}$ for inclusions and that the shape coefficient $I_{m}$ for the matrix can be deduced from the shape coefficients of the inclusions by (for nonzero wave vectors):

$$
I_{m}\left(\mathbf{k}_{(n)}\right)=-\sum_{\alpha \neq m} I_{\alpha}\left(\mathbf{k}_{(n)}\right) .
$$

For inclusion-matrix composites composed of two phases $I$ (inclusion) and $M$ (matrix), the recurrence relation (18) can then be written as

$$
\mathrm{e}^{i+1}=\mathrm{e}^{i}-\Gamma^{0} \cdot\left[\mathbf{L}_{M} \cdot \mathrm{e}^{i}+\left(\mathbf{L}_{I}-\mathbf{L}_{M}\right) \cdot\left(I_{I} * \mathbf{e}^{i}\right)\right] .
$$

The values of $I_{\alpha}$ are given below for inclusions having specific shapes. 
(a) Plane problems: Elliptic inclusions: The function $I_{\alpha}\left(\mathbf{k}_{(n)}\right)$ is given for elliptic inclusions within 2D problems by Nemat-Nasser and Hori (1999):

$$
I_{\alpha}\left(\mathbf{k}_{(n)}\right)=2 \cdot S_{\alpha} J_{1}(\eta) / \eta \cdot \mathrm{e}^{\mathrm{ik}(n) \cdot \mathbf{x}_{c}(\alpha)},
$$

where $J_{1}$ is the Bessel function of first kind and first order; $S_{\alpha}$ is the surface area of the inclusion; $\mathbf{x}_{c}(\alpha)$ is the vector position of the center of the inclusion $\alpha$.

$$
\eta=\left(k_{1(n)}^{2} a_{1}^{2}+k_{2(n)}^{2} a_{2}^{2}\right)^{1 / 2},
$$

where $a_{1}, a_{2}$ are the principal radii of the ellipse and $k_{1(n)}, k_{2(n)}$ are the components of $\mathbf{k}_{(n)}$ in the direction of the axes of the ellipse.

(b) Ellipsoidal inclusions: For ellipsoidal inclusions, the function $I_{\alpha}\left(\mathbf{k}_{(n)}\right)$ is given by Iwakuma and Nemat-Nasser (1983):

$$
I_{\alpha \cdot}\left(\mathbf{k}_{(n)}\right)=3 \cdot V_{\alpha} \cdot \frac{\sin (\eta)-\eta \cdot \cos (\eta)}{\eta^{3}} \cdot \mathrm{e}^{\mathrm{e}^{\mathbf{i}(n)} \cdot \mathbf{x}_{c}(\alpha)},
$$

where $V_{\alpha}$ is the volume of inclusion $\alpha ; \mathbf{x}_{c}(\alpha)$ is the vector position of the center of the inclusion $\alpha$.

$$
\eta=\left(k_{1(n)}^{2} a_{1}^{2}+k_{2(n)}^{2} a_{2}^{2}+k_{3(n)}^{2} a_{3}^{2}\right)^{1 / 2},
$$

where $a_{1}, a_{2}, a_{3}$ are the principal radii of the ellipsoid, $k_{1(n)}, k_{2(n)}, k_{3(n)}$ are the components of $\mathbf{k}_{(n)}$ in the direction of the axes of the ellipsoid.

Finally, the components of the effective elasticity tensor are obtained by keeping the Fourier component related to $\mathbf{k}=0$ of the local stress, which is equal, for a unit volume period, to the volumic mean of the local stresses.

\section{Comparison with previous solutions}

To check the validity of the previously described method, the solution for effective properties of a periodic fiber composite is now sought.

Let us consider a periodic fiber composite whose fibers have a circular cross-section and are periodically located, the centers being located along a squared lattice. Both phases are assumed isotropic. A "closed-form" solution of such a problem was given by Sabina et al. (2002) (in fact, the solution is obtained by adjusting the coefficients in an expansion of doubly periodic functions: Weierstrass functions and Natanzon functions). These authors recall that all elastic constants can be computed as soon as the following problems are solved:

(A) Plane strain problems: Assuming that the axis $x_{3}$ is oriented along the direction of the fibers, the three plane strain problems consist in Eqs. (1) (3) where the macroscopic plane deformation $\mathbf{E}$ is given by

$$
\begin{aligned}
& \text { PB1: } E_{i j}=\delta_{i 1} \delta_{j 1} . \\
& \text { PB2: } E_{i j}=\delta_{i 2} \delta_{j 2} . \\
& \text { PB3: } E_{i j}=\delta_{i 1} \delta_{j 2} .
\end{aligned}
$$

For a squared lattice and isotropic constituents, PB1 and PB2 are identical.

The solution of the previous section may be used by introducing convenient shape coefficients. These coefficients are given by (24) for $a_{1}=a_{2}=R$, where $R$ is the radius of 
the circular section of the fiber:

$$
I_{\alpha}(\mathbf{k})=2 \cdot \pi R \cdot J_{1}(k \cdot R) / k,
$$

where $\mathbf{k}=\mathbf{k}_{(n)}$ and $k=\left(k_{1}^{2}+k_{2}^{2}\right)^{1 / 2}=|\mathbf{k}|$, with

$$
k_{i}=k_{i(n)} \text {. }
$$

The value of the Green's tensor is given for an isotropic reference medium by Mura (1982):

$$
\begin{aligned}
& \Gamma_{i i i i}=4 A k_{i}^{2} / k^{2}-B k_{i}^{4} / k^{4}, \\
& \Gamma_{i i j j}=-B k_{i}^{2} k_{j}^{2} / k^{4}, \\
& \Gamma_{1212}=A-B k_{1}^{2} k_{2}^{2} / k^{4}, \\
& \Gamma_{i i i j}=2 A k_{i} k_{j} / k^{2}-B k_{i}^{3} k_{j} / k^{4},
\end{aligned}
$$

where $A=1 / 4 . \mu$ and $B=(\lambda+\mu) / \mu(\lambda+2 \mu)$.

(B) Antiplane problems: The antiplane problems correspond to degenerate scalar problems for which only the component of the displacement vector along $x_{3}$ is involved:

$$
\frac{\partial}{\partial x_{1}}\left(\mu(\mathbf{x}) \frac{\partial u_{3}}{\partial x_{1}}\right)+\frac{\partial}{\partial x_{2}}\left(\mu(\mathbf{x}) \frac{\partial u_{3}}{\partial x_{2}}\right)+\frac{\partial}{\partial x_{1}}\left(2 . \mu(\mathbf{x}) E_{31}\right)+\frac{\partial}{\partial x_{2}}\left(2 . \mu(\mathbf{x}) E_{32}\right)=0 .
$$

The two antiplane problems are therefore

PB4: $E_{i j}=\delta_{i 3} \delta_{j 1}$.

PB5: $E_{i j}=\delta_{i 3} \delta_{j 2}$.

For a squared lattice the solution of these two problems are identical.

The procedure is exactly the same as for the full elasticity problem, as soon as the following degenerate tensors are used:

$$
\begin{aligned}
& \Gamma_{3232}=A k_{2}^{2} / k^{2}, \\
& \Gamma_{3131}=A k_{1}^{2} / k^{2}, \\
& \Gamma_{3132}=A k_{1} k_{2} / k^{2} .
\end{aligned}
$$

Finally, for a squared lattice, only problems PB1, PB3 and PB4 need to be solved.

In this case, the solution of PB1 leads to the effective components: $L_{1111}^{\text {eff }}, L_{2222}^{\text {eff }}$, $L_{2211}^{\text {eff }}, L_{3322}^{\text {eff }}, L_{3311}^{\text {eff }}$.

The solution of PB3 leads to $L_{1212}^{\text {eff }}$ and the solution of PB4 leads to $L_{1313}^{\text {eff }}$ and $L_{2323}^{\text {eff }}$.

It is shown by Rodriguez-Ramos et al. (2001) that the solution of the localization problem for $E_{33}$ is a linear combination of the solutions to the problems related to $E_{11}$ and $E_{22}$. This leads to the obtaining of $L_{3333}^{\mathrm{eff}}$ as a linear combination of $L_{1111}^{\mathrm{eff}}$ and $L_{2222}^{\mathrm{eff}}$.

For that geometry, the effective elasticity tensor depends finally only on 6 constants. The transverse and longitudinal Young's moduli can then be obtained easily by inversion of the elasticity tensor.

The glass-epoxy composite studied by Meguid and Kalamkarov (1994), Luciano and Barbero (1994) and Sabina et al. (2002) is dealt with. The properties of the matrix are: 


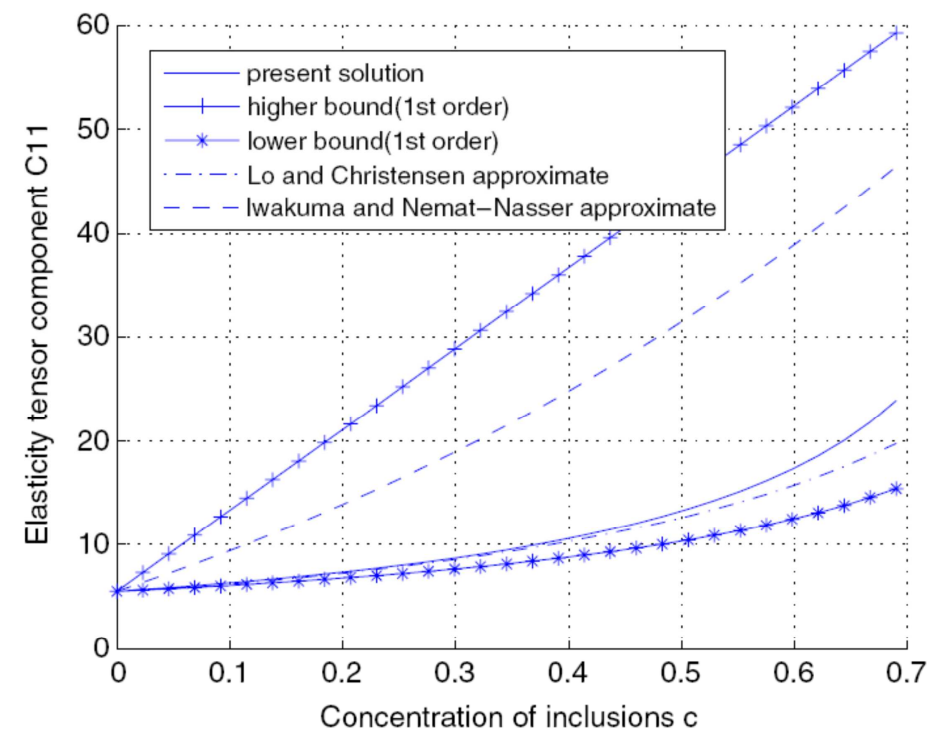

Fig. 1. Solution for transverse component $C_{11}=L_{1111}$. Comparison with approximate solutions and first order bounds.

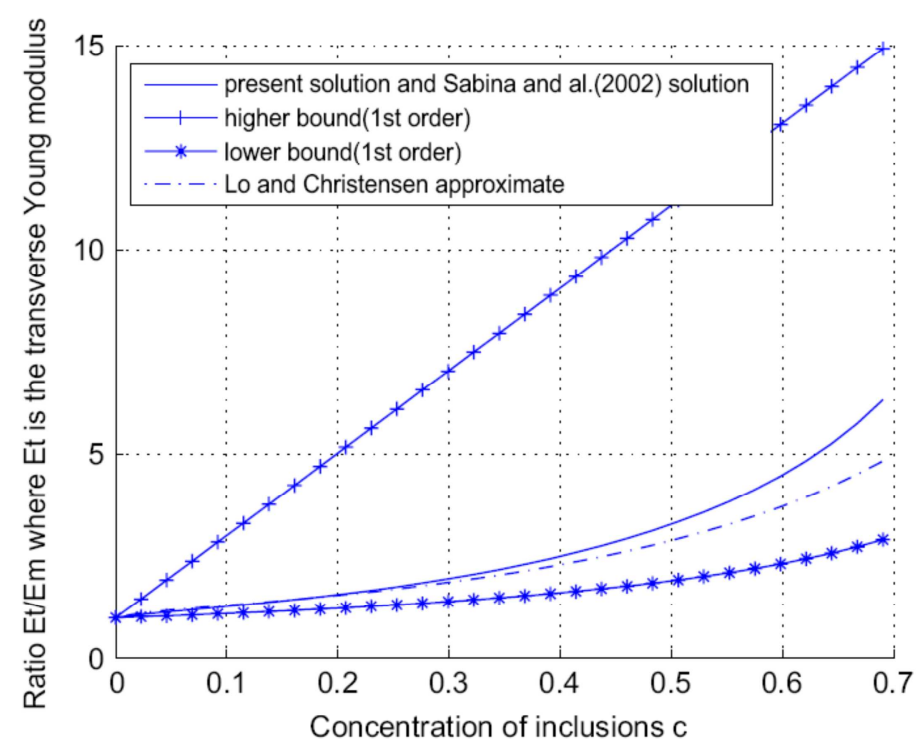

Fig. 2. Solution for transverse Young modulus. Comparison with first order bounds and Lo and Christensen solution.

$E_{M}=3.45 \mathrm{GPa}, v_{M}=0.35$ while the properties of the fibers are given by: $E_{I}=73.1 \mathrm{GPa}$, $v_{I}=0.22$.

Fig. 1 shows the values of $L_{1111}$ as a function of the fiber concentration, at convergence, compared to the Voigt and Reuss bounds and to the Christensen and Lo (1979) solution. An elastic comparison medium whose properties are given by $\mathbf{L}_{\text {ref }}=\left(\mathbf{L}_{M}+\mathbf{L}_{I}\right) / 2$ has been 
used to compute the solution. The value of the approximate solution of Iwakuma and Nemat-Nasser (1983) is also reported. The comparison with that approximate solution shows that this last one overevaluates the elastic coefficient.

Fig. 2 shows the values of the ratio $E_{\mathrm{t}} / E_{M}$ of the effective transverse modulus $E_{\mathrm{t}}$ to the Young's modulus of the matrix $E_{M}$. These values are identical to those obtained by Sabina et al. (2002).

\section{Convergence study}

The computation of the Neuman series depends on

- the quantity of wave numbers $N_{k}$ used within the different Fourier transforms;

- the number of terms $N_{s}$ kept within the Neuman series, which corresponds to the number of iterations used for the iterative process described in Section 3;

- the elastic properties of the elastic reference medium and of the phases.

\subsection{Convergence in wave numbers}

The effect of the quantity of wave numbers $N_{k}$ taken into account is studied in the case of the example used above. The error due to the iterative process can be evaluated by using the residue in the equilibrium equations (Michel et al., 1999) or more simply by using a direct comparison of the last term of the series compared to the sum at a given order (Eyre and Milton, 1999). In the following, the iterative process is stopped when the norm of the last term of the series is lesser than the norm of the sum of the series multiplied by a value $\alpha_{i t}$. Fig. 3 shows the variation of $L_{1111}$ for different values of $N_{k}$, number of wave vectors used for computing the different Fourier transforms, for $\alpha_{i t}=0.001$. It can be seen that

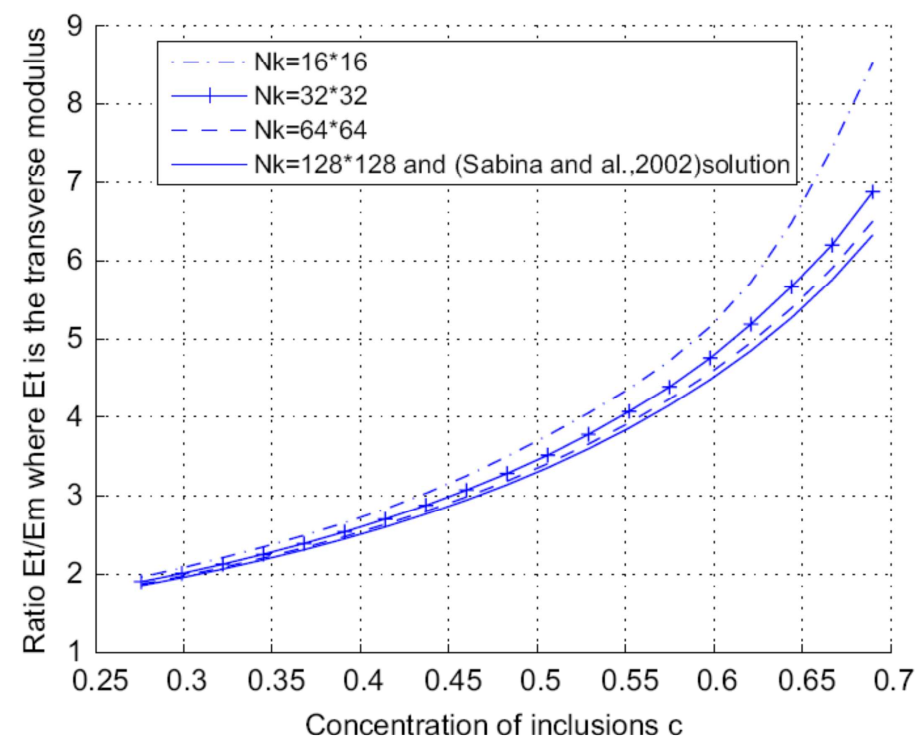

Fig. 3. Study of convergence in wave numbers. 
a number of wave vectors of 128 in each direction is convenient to reach the convergence. The values of the elastic coefficient computed by using increasing values of $N_{k}$ decreases continuously, showing that the values obtained constitute more and more precise upper bounds of $L_{1111}$.

The fact that successive results constitute upper bounds of the solution is due to the fact that the successive strain fields are compatible, their mean being equal to $\mathbf{E}$. The local stresses which are deduced from these strain fields lead therefore to upper bounds of the elasticity tensor.

\subsection{Optimization of the reference medium for a fiber-matrix material}

The convergence of the Neuman series and more precisely the effect of the properties of the reference medium on the convergence for given properties of the phases has been the subject of previous studies. An account of these studies can be found in Milton (2002). In this context, Eyre and Milton (1999) proposed an alternative recurrence scheme to accelerate the convergence rate.

Milton (2002) proposed for the optimal elasticity tensor to be used when building the Neuman series the value given by $\mathbf{L}_{M I}=\frac{1}{2}(\alpha+\beta) \mathbf{I}$, where $\mathbf{I}$ is the fourth-order identity tensor and $\alpha$ and $\beta$ are the maximum and the minimum eigenvalues of the elasticity tensors of the phases. A numerical study reported by Michel et al. (1999) for a mixture of two isotropic materials led to the use of an isotropic elasticity tensor whose properties are given by

$$
\lambda_{M O}=\frac{1}{2}\left(\lambda_{1}+\lambda_{2}\right) \text { and } \mu_{M O}=\frac{1}{2}\left(\mu_{1}+\mu_{2}\right) .
$$

Fig. 4 shows the number of iterations at convergence necessary when using the reference elasticity tensors $\mathbf{L}_{M I}$ and $\mathbf{L}_{M O}$ for the case of an uniaxial traction. It can be seen that the

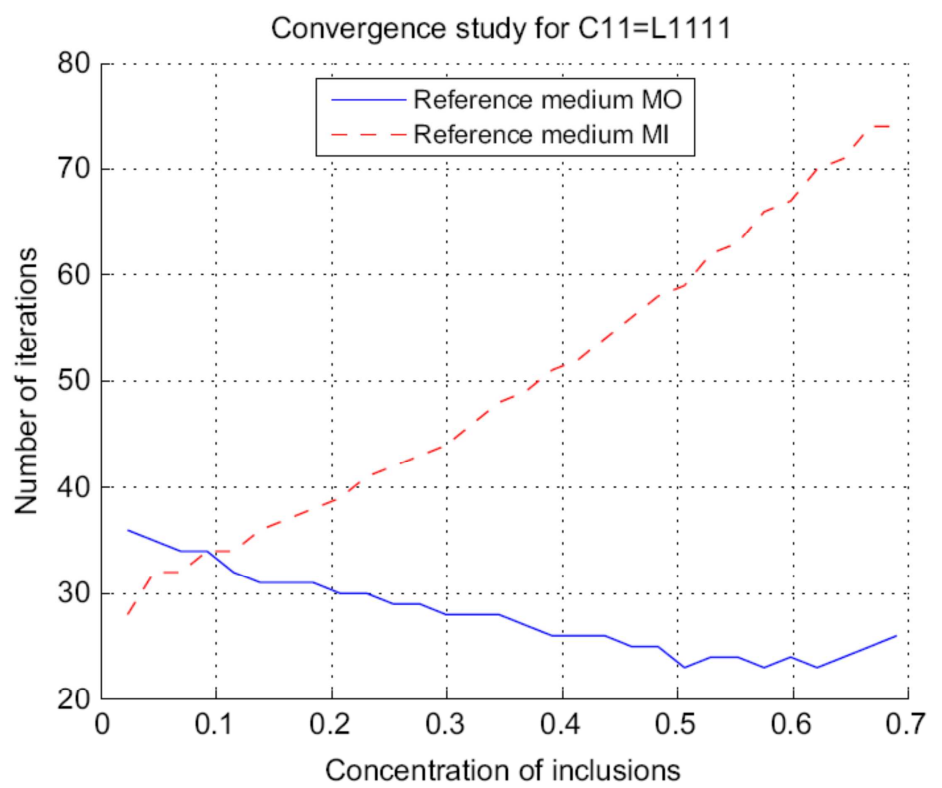

Fig. 4. Convergence of the iterative process when using previously proposed reference media. 
elasticity tensor $\mathbf{L}_{M O}$ gives better results. This result seems to be due to the fact that the identity elasticity tensor used in $\mathbf{L}_{M I}$ corresponds to a vanishing value of the Poisson's ratio, which does not seem optimal to compute the effective elasticity tensor for a mixture of two media having a non-zero Poisson's ratio.

From a general point of view, the convergence rate is an increasing function of the contrast between the stiffnesses of both materials. The convergence rate depends also on the choice of the reference elasticity tensor. In order to study the influence of the properties of the elastic reference medium on the fastness of convergence, the following procedure has been used: in a first step, the Poisson's ratios of the matrix and of the fibers are assumed identical and the ratio between the Young's moduli of both phases were varied between $E_{I} / E_{M}=0.1$ and $E_{I} / E_{M}=1000$. The reference elasticity tensor is assumed to be of the form:

$$
\mathbf{L}_{\text {ref }}=\alpha \mathbf{L}_{M}+(1-\alpha) \mathbf{L}_{I},
$$

where $\alpha$ is taken between 0 and 1 . In a second step, the effect of the differences between Poisson's ratios will be studied.

Table 1 shows the results obtained for stiffer inclusions, inclusion and matrix having a Poisson's ratio equal to 0.3 . Each table displays the optimal value of $\alpha$ and the number of terms $N$ kept within the series to insure the convergence. In comparison, the number of terms necessary when using $\alpha=0.5$ as suggested by Michel et al. (1999) is also given. The optimal value of $\alpha$ is comprised between 0.4 and 0.45 for all the concentrations and contrasts studied. The use of $\alpha=0.4$ for each case leads practically to nearly the same number of iterations as the one obtained for the optimal value of $\alpha$, as shown by the last column of the table. The comparison between the values of $N$ obtained for the optimal value of $\alpha$ and for $\alpha=0.5$ shows that the use of the optimal value of $\alpha$ leads to a gain on the convergence rate (compared to $\alpha=0.5$ ) which can reach nearly 20 for the higher contrast. In fact, the number of iterations increases drastically when $\alpha$ is near to 0.5 . In addition, it can be seen that the number of terms needed to reach convergence is proportional to $N$ when $\alpha=0.5$, when it increases as $N^{p}, p$ being between 0.4 and 0.5 for the optimal value of $\alpha$. It seems that using a convenient value of $\alpha$ leads to the same kind of convergence as the "accelerated" scheme described in Eyre and Milton (1999).

A systematic comparison between the same contrasts on the Young's moduli but for the case of a Poisson's ratio equal to 0.4 has been performed and has shown that the optimal

Table 1

Convergence study for stiffer inclusions and $v_{I}=v_{M}=0.3$

\begin{tabular}{lllccc}
\hline Moduli ratio & $c=V_{I} / V$ & Optimal $\alpha$ & $N_{\text {opt }}$ & $N(\alpha=0.5)$ & $N(\alpha=0.4)$ \\
\hline \multirow{2}{*}{$E_{I} / E_{M}=10$} & 0.2 & 0.4 & 7 & 10 & 7 \\
& 0.4 & 0.4 & 9 & 9 & 9 \\
$E_{I} / E_{M}=100$ & 0.6 & 0.45 & 11 & 11 & 12 \\
& 0.2 & 0.4 & 17 & 82 & 17 \\
$E_{I} / E_{M}=1000$ & 0.4 & 0.45 & 19 & 63 & 20 \\
& 0.6 & 0.45 & 29 & 48 & 31 \\
\hline
\end{tabular}


value of $\alpha$ is practically not affected by the Poisson's ratio (assumed equal for matrix and inclusion) within the range $[0.3,0.4]$.

Table 2 shows the results obtained for inclusions softer than the matrix and for voids, inclusion and matrix having a Poisson's ratio equal to 0.3 . The optimal values of $\alpha$ are now located between 0.5 and 0.7. As previously, a value of $\alpha=0.5$ is not optimal for higher concentrations for which a value of $\alpha$ between 0.65 and 0.7 is largely better, the gain attaining nearly 10 . The last column shows that a constant value of $\alpha=0.65$ is nearly optimal and should be therefore recommended for studying a medium with softer inclusions and voids. Finally, in the case of voids, there is no convergence difficulties, such as those found by authors which used the space discretization and a numerical procedure to solve the equilibrium equation.

The previous study was performed by using the same Poisson's ratio for both components. The case of inclusions and matrix having different Poisson's ratios has been also studied.

Table 3 shows the results for $E_{I} / E_{M}=100$ and $E_{I} / E_{M}=0.1$ and different Poisson's ratios for the phases. The results show that the convergence is nearly the same when using a value $\alpha=0.4$ for the first case and $\alpha=0.65$ for the second case. When using a value $\alpha=0.5$, the convergence rate is lower than for the case where Poisson's ratios are the same.

Finally, it can be concluded that (for $v$ being within [0.2,0.4]): for fibers stiffer than the matrix, a value $\alpha=0.4$ can be used in any case, leading to nearly the best convergence rate.

Table 2

Convergence study for softer inclusions and $v_{I}=v_{M}=0.3$

\begin{tabular}{llllll}
\hline Moduli ratio & $c=V_{I} / V$ & Optimal $\alpha$ & $N_{\text {opt }}$ & $N(\alpha=0.5)$ & $N(\alpha=0.65)$ \\
\hline \multirow{3}{*}{$E_{I} / E_{M}=0.1$} & 0.2 & 0.5 & 5 & 5 & 6 \\
& 0.4 & 0.65 & 5 & 8 & 5 \\
& 0.6 & 0.65 & 6 & 13 & 6 \\
Voids & 0.2 & 0.6 & 6 & 9 & 7 \\
& 0.4 & 0.65 & 6 & 22 & 6 \\
& 0.6 & 0.7 & 6 & 65 & 7 \\
\hline
\end{tabular}

Table 3

Convergence study for $v_{I}=0.4$ and $v_{M}=0.2$

\begin{tabular}{llllll}
\hline Moduli ratio & $c=V_{I} / V$ & Optimal $\alpha$ & $N_{\text {opt }}$ & $N(\alpha=0.5)$ & $N(\alpha=0.4)$ \\
\hline \multirow{2}{*}{$E_{I} / E_{M}=100$} & 0.2 & 0.4 & 22 & 184 & 22 \\
& 0.4 & 0.45 & 26 & 145 & 27 \\
Moduli ratio & 0.6 & 0.45 & 41 & 126 & 44 \\
\hline \multirow{2}{*}{$E_{I} / E_{M}=0.1$} & $c=V_{I} / V$ & Optimal $\alpha$ & $N_{\text {opt }}$ & $N(\alpha=0.5)$ & $N(\alpha=0.65)$ \\
& 0.2 & 0.65 & 5 & 10 & 5 \\
& 0.4 & 0.65 & 5 & 14 & 5 \\
\hline
\end{tabular}


For fibers softer than the matrix, a value $\alpha=0.65$ leads to nearly the best convergence rate, and can be used for empty fibers.

\section{The limit cases}

\subsection{Empty fibers}

The case of infinite contrast between inclusion and matrix, including voids, is generally considered as not being solvable when using the Neuman series (as quoted for example by Michel et al., 1999) when the numerical (non-exact) procedure for computing the local stress is used. As shown previously, there is no convergence difficulty when using a convenient reference elasticity tensor and exact values of the shape factors. The case of empty fibers converges quickly. To check the validity of the present solution for empty fibers, the computation was performed for the case studied by Sabina et al. (2002). Fig. 5 shows the values obtained for the transverse Young's modulus, which comply with the results obtained previously by these authors.

\subsection{Rigid fibers}

The case of rigid fibers has been solved for fibers with a circular cross-section by Sabina et al. (2002). It is obvious that the series (10) cannot be used when the elastic moduli of the inclusion tend to infinity. Previous studies led to reach the solution for rigid fibers as the limit when the contrast between inclusion and matrix becomes very large. Recent results used for example a Lagrangian augmented method to deal with the case of rigid inclusions (Michel et al., 2000). As shown above, the number of iterations to be used increases indeed when the inclusions become stiffer, especially when the elasticity tensor of the reference

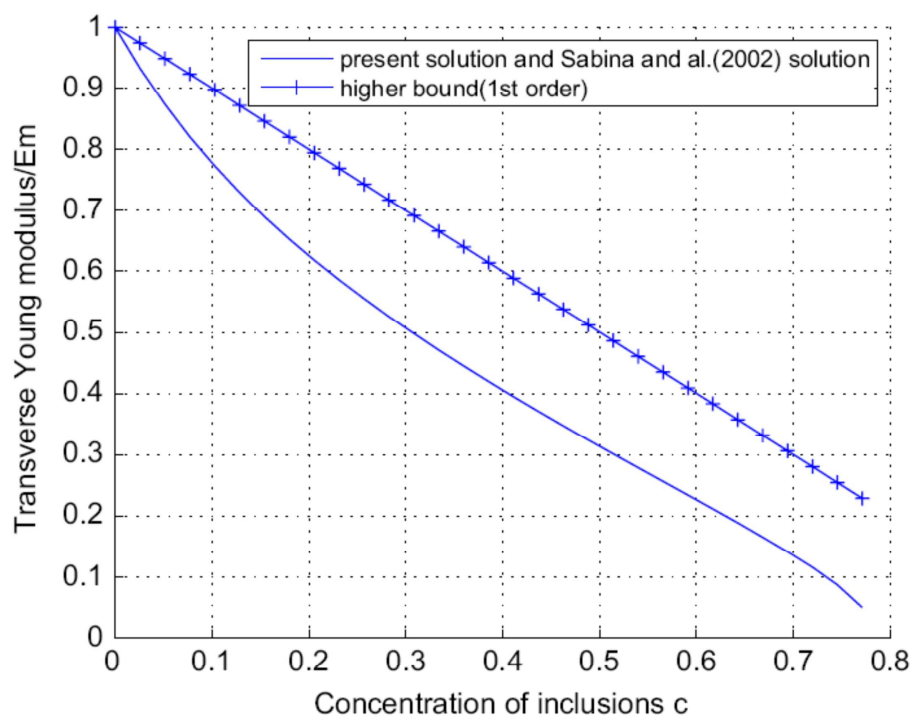

Fig. 5. Variation of Young's modulus vs the concentration of fibers for empty fibers (voids). 
medium is not optimized. It is, however, natural in this case to prefer a formulation based on the compliance tensor $\mathbf{M}(\mathbf{x})$ instead of the elasticity tensor.

The classical integral equation in e recalled above in Eq. (9) is the basis of the solution of the periodic elasticity problem. It can be shown (Kröner, 1971) that this integral equation is equivalent to an integral equation in the stress tensor $\boldsymbol{\sigma}$ written as

$$
\boldsymbol{\sigma}(\mathbf{x})=\mathbf{\Sigma}-\Delta_{0} *(\delta \mathbf{M} . \boldsymbol{\sigma}),
$$

where $\mathbf{\Sigma}$ is the macroscopic stress tensor, equal to the volumic mean stress.

$$
\delta \mathbf{M}(\mathbf{x})=\mathbf{M}(\mathbf{x})-\mathbf{M}_{0}, \quad \mathbf{M}_{0}=\mathbf{L}_{0}^{-1} \quad \text { and } \quad \mathbf{M}(\mathbf{x})=\mathbf{L}(\mathbf{x})^{-1} .
$$

$\boldsymbol{\Delta}_{0}$ is the Green's tensor for stresses, obtained from the reference elasticity tensor $\mathbf{L}_{0}$ and from the Green's tensor for deformation $\Gamma_{0}$ by

$$
\Delta_{0}=\mathbf{L}_{0}-\mathbf{L}_{0} \cdot \Gamma_{0} \cdot \mathbf{L}_{0} .
$$

The components of $\Delta_{0}$ are thus given for the components used to study the case of fibers by

$$
\begin{aligned}
& \Delta_{i i i i}=\lambda+2 \mu-\lambda^{2} \Gamma_{l l l l}-4 \mu(\lambda+\mu) \Gamma_{i i i i}-2 \lambda(\lambda+2 \mu) \Gamma_{1122}, \\
& \Delta_{1122}=\lambda-\Gamma_{l l l l} \lambda(\lambda+2 \mu)-2 \Gamma_{1122}\left(\lambda^{2}+2 \lambda \mu+2 \mu^{2}\right), \\
& \Delta_{i i 12}=-2 \lambda \mu \Gamma_{k k 12}-4 \mu^{2} \Gamma_{i i 12}, \\
& \Delta_{1212}=\mu-4 \mu^{2} \Gamma_{1212}, \\
& \Delta_{3 i 3 j}=\mu-4 \mu^{2} \Gamma_{3 i 3 j}, \\
& \Delta_{3132}=-4 \mu^{2} \Gamma_{3132} .
\end{aligned}
$$

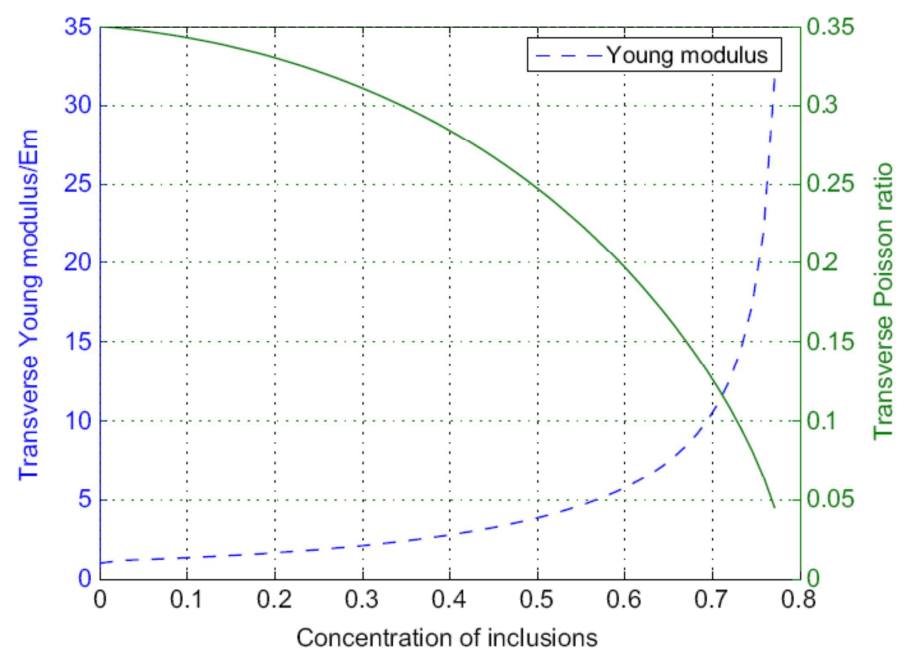

Fig. 6. Variation of the transverse Young's modulus and Poisson's ratio for a composite with rigid inclusions. 
The computation of the local stress can be effected as previously by expansion into the Neuman series, or in an equivalent way by the recurrence process:

$$
\boldsymbol{\sigma}^{i+1}=\boldsymbol{\Sigma}-\Delta_{0} *(\delta \mathbf{M} . \boldsymbol{\sigma})^{i} .
$$

The computation is then strictly identical to the computation described within Section 3, using, for two-phase problems the recurrence relation:

$$
\boldsymbol{\sigma}^{i+1}=\boldsymbol{\Sigma}-\boldsymbol{\Delta}_{0} *\left[\left(\mathbf{M}_{M}-\mathbf{M}_{0}\right) \cdot \boldsymbol{\sigma}^{i}+\left(\mathbf{M}_{I}-\mathbf{M}_{M}\right) \cdot\left(I_{I} * \boldsymbol{\sigma}^{i}\right)\right] .
$$

Fig. 6 shows the values of the transverse modulus $E_{\mathrm{t}}$ and of the transverse Poisson's ratio for the case of rigid fibers, the matrix having a Poisson's ratio equal to 0.35 , and the number of wave numbers being equal to 128 along both directions. These results comply with the results obtained by (Sabina et al., 2002).

In addition, it is worthwhile to mention that the results obtained when using small values of $N_{k}$ lead to lower bounds of the effective Young's modulus. When the contrast is not infinite, it is therefore possible to use either the "strain solution" corresponding to Eq. (14) or the "stress solution" corresponding to Eq. (36). For a given quantity of wave numbers, the solutions lead to upper and lower bounds of the effective properties.

\section{Application for new configurations}

The method previously described can be adapted to many new configurations. The examples below show the application to two new configurations: the case of elliptic fibers and the case of multi-phase fibrous elastic composites.

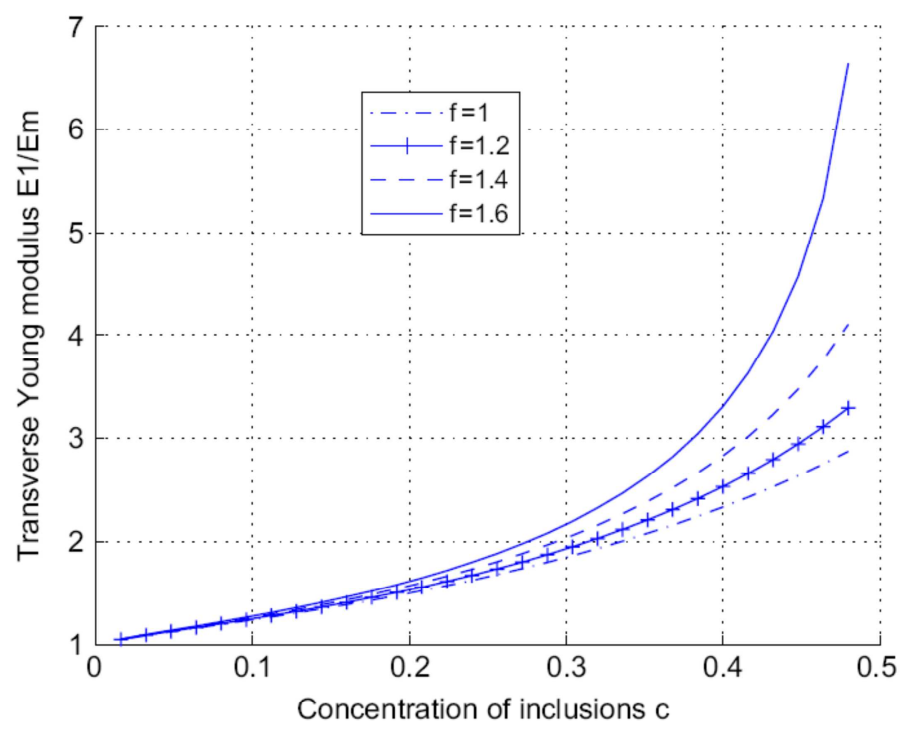

Fig. 7. Transverse Young's modulus in the direction of $x_{1}$ for fibers with an elliptic cross-section as a function of the concentration and of the ratio of the axes of the ellipse $f=a_{1} / a_{2}$. 


\subsection{Composites with elliptic fibers}

The exact solution for effective properties of composites with ellipsoidal inclusions has been obtained by Kushch (1997). The results obtained by Iwakuma and Nemat-Nasser (1983) can be applied for the case of fibers with elliptical cross-sections, giving approximate solutions, but it does not seem that exact solutions have been obtained up to now for the case of fibers with elliptic cross-sections.

The solution developed in the present paper can easily be used for composites with elliptic fibers, by using the shape coefficient given in Eq. (24). Fig. 7 shows the different values of the transverse Young's modulus for different kinds of elliptical fibers within a squared lattice. The elliptic section, whose principal axis is oriented along the axis $x_{1}$ is characterized by the ratio $f=a_{1} / a_{2}$. The result shows the increase of the transverse Young's modulus when the ratio $f=a_{1} / a_{2}$ increases, which is physically satisfying.

\subsection{Multi-phase fibrous elastic composites}

The variation of properties around the fibers may be taken into account by using multiphase fibrous elastic composites, as shown by example by Guinovart-Diaz et al. (2005) for cylindrical fibers. An example of such a situation is shown in Fig. 8.

This kind of situation can account for example for a diminution of the elastic properties of the matrix near the fiber due to a localized damage. The method described in the present paper is able to take into account such situations by using suitable shape factors. As shown in Fig. 8, the composite material is now composed of three phases.

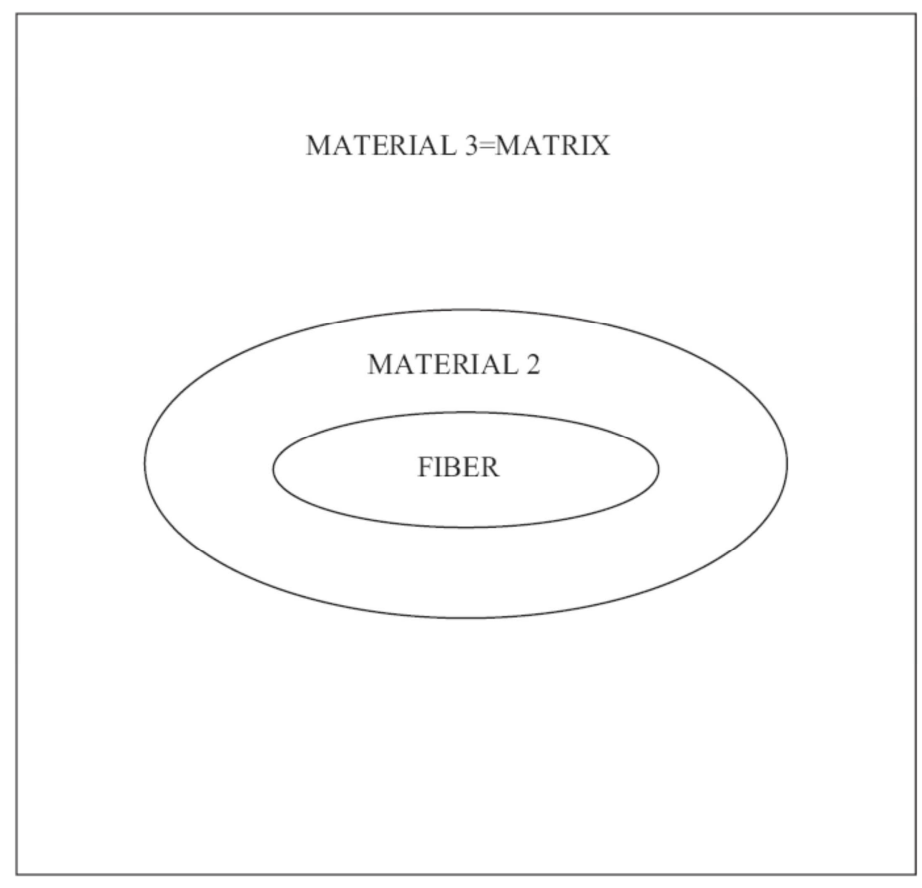

Fig. 8. Section of a period for a multi-phase fibrous composite. 


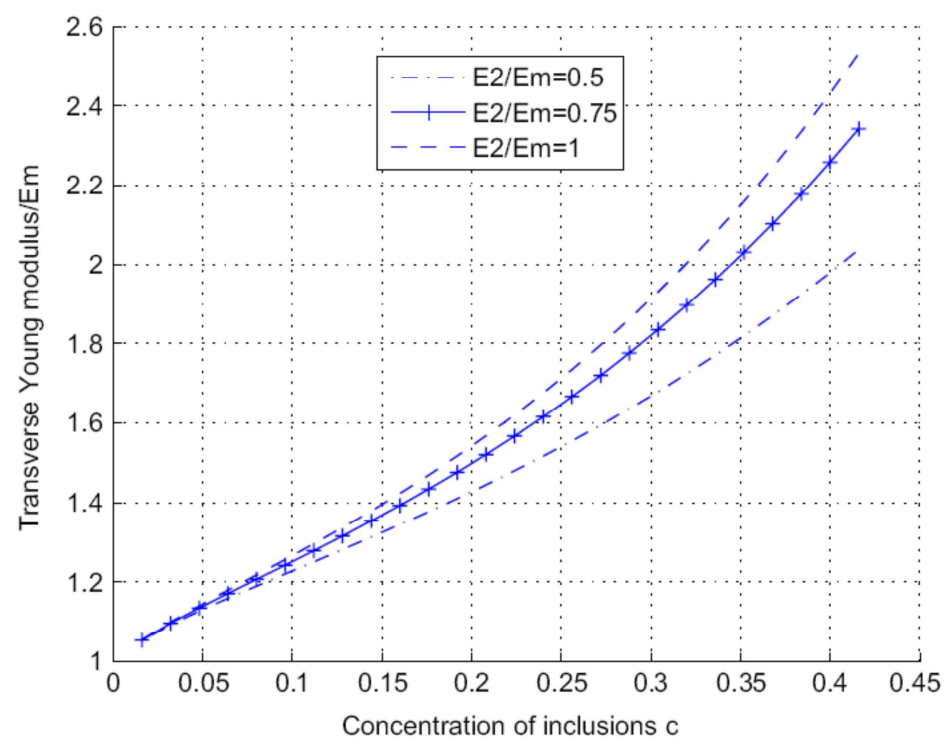

Fig. 9. Variation of the Young's modulus as a function of the concentration for different ratios $E_{2} / E_{\mathrm{m}}, E_{2}$ being the Young's modulus of the intermediate zone.

Material 1 constitutes the fiber, while material 2 constitutes the medium near the fiber and material 3 constitutes the matrix. As shown previously, the method allows to take into account fibers with an elliptic cross-section. The shape factors for material 1 is given for an elliptic section defined by the half axes of the ellipse $\left(a_{1}, a_{2}\right)$ by Eq. (24), while the shape factors for material 2, located between the ellipse having the axes $a_{1}, a_{2}$ and the ellipse having the axes $b_{1}, b_{2}$ are given by

$$
I_{\alpha}\left(\mathbf{k}_{(n)}\right)=2 \cdot S_{\alpha b} J_{1}\left(\eta_{b}\right) / \eta_{b} \cdot \mathrm{e}^{\mathbf{i} \mathbf{k}_{(n)} \cdot \mathbf{x}_{c}(\alpha)}-2 \cdot S_{\alpha} J_{1}(\eta) / \eta \cdot \mathrm{e}^{\mathrm{i} \mathbf{k}_{(n)} \cdot \mathbf{x}_{c}(\alpha)},
$$

where $\eta_{b}=\left(k_{1}^{2} b_{1}^{2}+k_{2}^{2} b_{2}^{2}\right)^{1 / 2}$ and $S_{\alpha b}=\pi b_{1} b_{2}$.

The values of the transverse modulus is given in Fig. 9 for the situation where $a_{1} / a_{2}=$ $b_{1} / b_{2}=1$ (circular boundaries between phases) and $b_{1} / a_{1}=1.1$, taking into account the following properties of materials $1-3$ :

Material 1: identical to the example of Section 4.

Material 2: Poisson's ratio $v$ identical to the one of the fiber and Young's modulus $E_{2}=$ $\beta . E_{\mathrm{m}}$ where $E_{\mathrm{m}}$ is the Young's modulus of the matrix, $\beta$ being a factor smaller than 1 .

Material 3: identical to the matrix in Section 4.

Fig. 9 shows the variation of the transverse Young's modulus as a function of the concentration, for different values of the contrast $\beta$ between materials 1 and 2 .

The result shows that even for a thickness of the softer layer along the fiber equal to $0.1 a_{1}, a_{1}$ being the radius of the fiber, a significant reduction of the Young's modulus can be observed, when the concentration of the inclusions increases. 


\section{Synthesis and conclusion}

The paper has described an explicit series solution to obtain the effective properties of composites having periodically located heterogneities. The method of solution uses the classical expansion along Neuman series of the solution of the periodic elasticity problem in Fourier space, based on the Green's tensor and exact expressions of the shape factors in Fourier space. The solutions related to different numbers of wave numbers kept in the Fourier series constitute bounds of the solution. Some properties of convergence of the solution were presented, more specifically concerning the elasticity tensor of the reference medium. A comparison was effected with previous exact solutions for a fiber composite made of cylindrical fibers with circular cross-sections and with previous estimates. The comparison is made mostly with the results obtained by Sabina et al. (2002). In comparison, the present paper gives an explicit solution, while the solution obtained by Sabina et al. (2002) needs to solve a linear infinite system of equations to obtain the coefficients of the expansion along suitable doubly periodic functions. The main advantage of the present method is its great flexibility, as shown by the different examples presented for new situations concerning the study of fiber composites: composites with elliptic crosssections and multi-phase fibrous composites. The method can be extended to the case where the components are made of elastic anisotropic materials. In the future, the method described in the present paper will be compared with other iterative methods for inclusionmatrix composites, such as the accelerated iterative scheme of Eyre and Milton (1999) or the fast multipole method.

\section{References}

Adams, D., Doner, D., 1967. Transverse normal loading of a unidirectional composite. J. Comput. Math. 1, 152. Auriault, J., Bonnet, G., 1985. Dynamique des composites élastiques périodiques. Arch. Mech. 37 (4-5), 269-284. Bensoussan, A., Lions, J., Papanicolaou, G., 1978. Asymptotic Analysis for Periodic Structures. North-Holland, Amsterdam.

Boutin, C., 1996. Microstructural effects in elastic composites. Int. J. Eng. Sci. 33 (7), 1023-1051.

Brown, W., 1955. Solid mixture permittivities. J. Comput. Math. 23, 1514-1517.

Christensen, R., Lo, K., 1979. Solutions for effective shear properties in three phase sphere and cylinder models. J. Mech. Phys. Solids 27, 315-330.

Cohen, I., 2004. Simple algebraic approximations for the effective elastic moduli of cubic arrays of spheres. J. Mech. Phys. Solids 52, 2167-2183.

Eyre, D., Milton, G., 1999. A fast numerical scheme for computing the response of composites using grid refinement. Eur. Phys. J. Appl. Phys. 6, 41-47.

Guinovart-Diaz, R., Bravo-Castillero, J., Rodriguez-Ramos, R., Sabina, F., 2001. Closed-form expressions for the effective coefficients of fibre-reinforced composite with transversely isotropic constituents. J. Mech. Phys. Solids 49, 1445-1462.

Guinovart-Diaz, R., Bravo-Castillero, J., Rodriguez-Ramos, R., Sabina, F., Otero-Hernandez, J., Maugin, G., 2005. A recursive asymptotic homogenization scheme for multi-phase fibrous elastic composites. Mech. Mater. $37,1119-1131$.

Gusev, A., 1997. Representative volume element size for elastic composites: a numerical study. J. Mech. Phys. Solids 45 (7), 1449-1459.

Hashin, Z., Shtrikman, S., 1962. On some variational principles in anisotropic and nonhomogeneous elasticity. J. Mech. Phys. Solids 10, 335-342.

Helsing, J., 1995. An integral equation method for elastostatics of periodic composites. J. Mech. Phys. Solids 43 (6), 815-828.

Iwakuma, T., Nemat-Nasser, S., 1983. Composites with periodic microstructure. Comput. Struct. 16 (1-4), 13-19. 
Kantor, Y., Bergman, D., 1982. Elastostatic resonances. a new approach to the calculation of the effective elastic constants of composites. J. Mech. Phys. Solids 30 (5), 355-376.

Kröner, E., 1971. Statistical Continuum Mechanics. Springer-Verlag, Wien.

Kushch, V., 1997. Microstresses and effective elastic moduli of a solid reinforced by periodically spheroidal particles. Int. J. Solids Struct. 34 (11), 1353-1366.

Luciano, R., Barbero, E., 1994. Formulas for the stiffness of composites with periodic microstructure. Int. J. Solids Struct. 31 (21), 2933-2944.

Meguid, S., Kalamkarov, A., 1994. Asymptotic homogenization of elastic composite materials with a regular structure. Int. J. Solids Struct. 31, 303-316.

Michel, J., Moulinec, H., Suquet, P., 1999. Effective properties of composite materials with periodic microstructure: a computational approach. Comput. Meth. Appl. Mech. Eng. 172, 109-143.

Michel, J., Moulinec, H., Suquet, P., 2000. A computational method based on Lagrangians and fast Fourier transforms for composites with high contrast. Comput. Model. Eng. Sci. 1 (2), 79-88.

Milton, G., 2002. The Theory of Composites. Cambridge University Press, Cambridge.

Moulinec, H., Suquet, P., 1994. A fast numerical method for computing the linear and nonlinear properties of composites. C. R. Acad. Sci. II 318, 1417-1423.

Mura, T., 1982. Micromechanics of Defects in Solids. Martinus-Nijhoff, Dordrecht.

Nemat-Nasser, S., Hori, M., 1999. Micromechanics: Overall Properties of Heterogeneous Materials. NorthHolland, Amsterdam.

Rodriguez-Ramos, R., Sabina, F., Guinovart-Diaz, R., Bravo-Castillero, J., 2001. Closed-form expressions for the effective coefficients of a fiber-reinforced composite with transversely isotropic constituents. I. Elastic and square symmetry. Mech. Mater. 33 (4), 223-235.

Sabina, F., Bravo-Castillero, J., Guinovart-Diaz, R., Rodriguez-Ramos, R., Valdiviezo-Mijangos, O., 2002. Overall behaviour of two-dimensional periodic composites. Int. J. Solids Struct. 39, 483-497.

Sanchez-Palencia, E., 1974. Comportement local et macroscopique d'un type de milieux physiques hétérogenes. Int. J. Eng. Sci. 12, 331-351.

Sanchez-Palencia, E., 1980. Nonhomogeneous Media and Vibration Theory, Lecture Notes in Physics, vol. 127. Springer, Berlin.

Sangani, A., 1987. Elastic coefficients of composites containing spherical inclusions in a periodic array. J. Mech. Phys. Solids 35, 1-21.

Segurado, J., Llorca, J., 2002. A numerical approximation to the elastic properties of sphere-reinforced composites. J. Mech. Phys. Solids 50, 2107-2121. 